\title{
Ocular injuries - a review
}

\section{VN Sukati}

Discipline of Optometry, School of Physiotherapy, Sport Science and Optometry, University of KwaZulu-Natal, Westville Campus, Private Bag X54001, Durban, 4000 South Africa

Received 28 January 2012; revised version accepted 30 May 2012

<mavegy@gmail.com>

\section{Introduction}

All ocular structures are vulnerable to injury, but the site often depends on the cause and mechanism of ocular injury ${ }^{1}$. The anterior segment of the eye which consists of the cornea, conjunctiva, trabecular meshwork, anterior chamber, iris, and crystalline lens is vulnerable to direct trauma. Posterior ocular structures include the retina, choroid and optic nerve ${ }^{1}$. The worst outcome is often seen in the combined anterior and posterior segment injuries with the possibility of losing all useful vision ${ }^{2-4}$. Ocular injuries are divided into open globe and closed globe injuries, however, there may be an overlap in their classification based on the causative agent or inflicting object involved. An open globe injury (an injury penetrating into the globe) involves a full thickness wound of the corneoscleral wall which may result from penetrating or blunt eye trauma. Open globe injuries include lacerations which are futher divided into penetrating injuries, perforating injuries and intraocular foreign bodies (Figure 1). Closed globe injuries are commonly due to blunt trauma whereby the corneoscleral wall of the globe remains intact (a partial thickness corneal wound) however, intraocular damage may be present. They are divided into burns, blunt trauma/contusions and lamellar lacerations. Ruptures are caused by blunt objects with the actual wound being produced by an inside-out mechanism. If the inflicting object is blunt, it can result in either a contusion or a rupture (open globe) $)^{5-8}$.

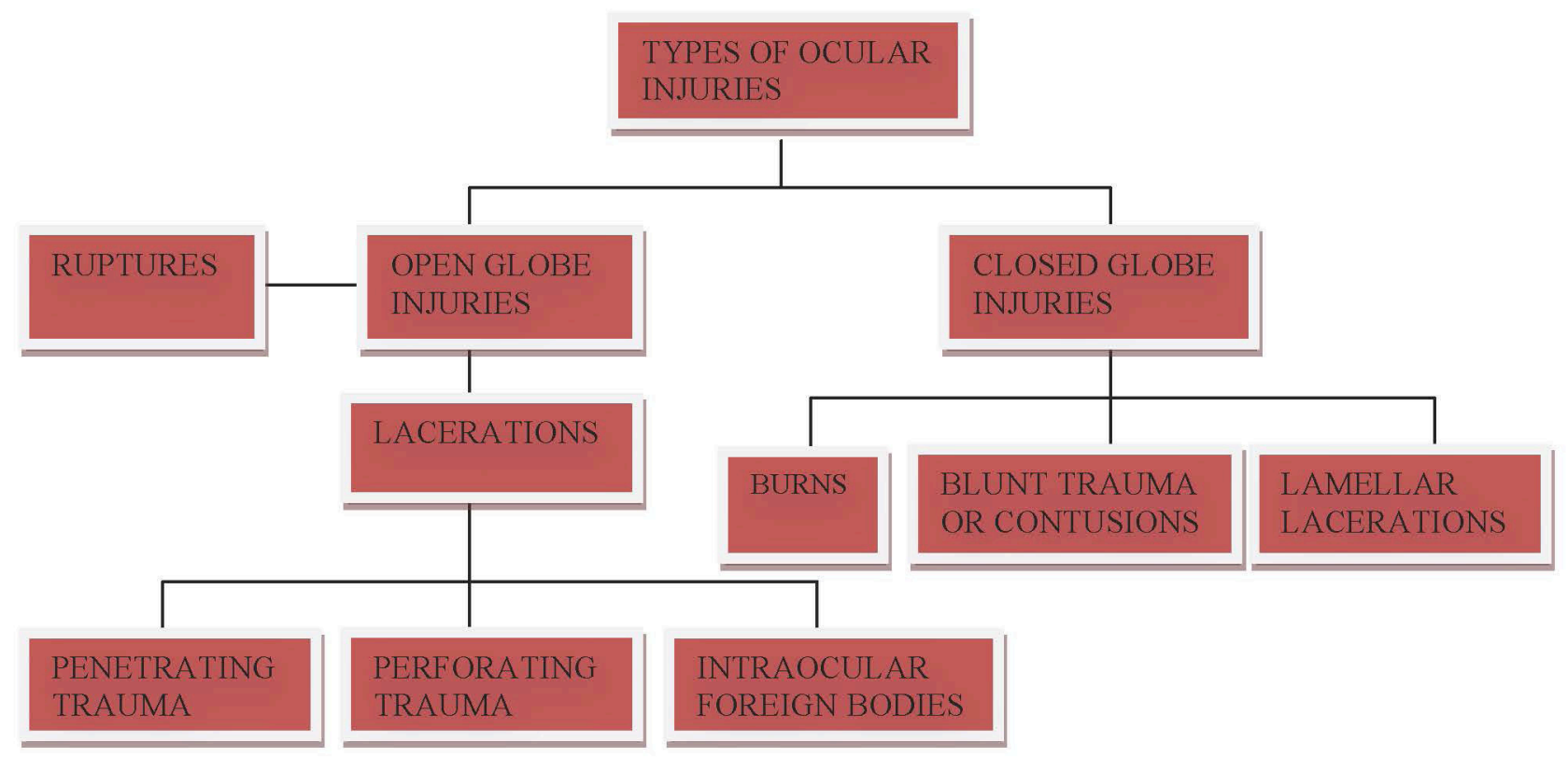

Figure 1: Standardized classification of ocular trauma using the Birmingham Eye Trauma Terminology (BETT) classification (Kuhn et al, 1996). 
Open and closed globe injuries are further described in terms of zones, elaborating on which structure of the eye the wound involves and to what extent it is. For open globe injuries a zone I wound involves the cornea, a zone II wound extends into the anterior $5 \mathrm{~mm}$ of the sclera and a zone III wound involves the sclera extending more than $5 \mathrm{~mm}$ from limbus. In the case of closed globe injuries, a zone I wound involves only the conjunctiva, sclera or cornea, a zone II injury includes the anterior chamber including the lens and zonules and a zone III injury involves posterior structures including the vitreous, retina, optic nerve, choroid and ciliary body 6,7 . In the past, there was no existence of a standardized classification of injuries despite the growth of interest in the publication of studies on eye injuries. However, recent studies approve the existence of the new classification which has provided better diagnoses and management of these injuries by eye health practitioners ${ }^{6,8,9}$.

\section{Open globe injuries}

\section{Lacerations}

"A laceration is a full thickness wound of the eye wall, usually caused by a sharp object. The wound occurs at the impact site by an outside-in mechanism. The classification is based on whether an intra-ocular foreign body or an exit wound is also present"6. Occasionally, an exit wound may be created by the object while remaining partially intraocular ${ }^{6}$.

Lacerations to the eyelids and the conjunctiva commonly occur from sharp objects but can also occur from a fall ${ }^{10}$. Most corneoscleral lacerations are caused by glass from shattered spectacles and broken windows and associated with blunt trauma of flying objects ${ }^{4}, 10$. Lacerations may occur in one of two ways: i) lacerations without prolapse of tissue when the eyeball has been penetrated anteriorly but without prolapse of the intra-ocular contents; and ii) lacerations with prolapse when a small portion of iris prolapses through a wound, or uveal tissue has been injured. Corneal lacerations can involve the iris and crystalline lens forming a cataract whereby management depends on the duration and extent of the incarceration ${ }^{11,12}$. Corneal lacerations frequently result in prolapse of the iris with distortion of the pupil. Hyphaema is often present reducing vision in the affected eye ${ }^{11,13}$.
Ocular lacerations are treated in different ways depending on whether or not there is tissue prolapse. However, the surgeon has to explore the extent of the wound first and then determine the status of the crystalline lens whether to remove it or not with the aid of a slit lamp. A crystalline lens can only be removed following a water tight closure of the laceration. If the wound is extensive and loss of intra-ocular contents has been great enough and the prognosis for useful function is hopeless, enucleation/evisceration is indicated as a primary surgical procedure ${ }^{14-16}$. However, when the wound is clean without tissue prolapse and free from contamination, it can usually be repaired by direct interrupted sutures and can often heal spontaneously with the aid of an eye pad, contact lenses, or cyanoacrylate adhesives while administering a topical antibiotic and controlling the patient's pain with oral analgesics. A precaution must be taken for a self sealing wound because when an edematous cornea subsides a wound leak may develop. Therefore, Seidel test is indicated for evaluation of a corneal wound leak to determine whether aqueous is being emitted or not $^{14,15}$. The patient needs to be referred to the nearest ophthalmologist for surgical repair as soon as possible to restore the anatomy or structural integrity of the globe irrespective of the extent of the injury and the initial visual acuity. If a delay in specialist care is anticipated, a systemic oral antibiotic and tetanus prophylaxis should be administered to avoid development of endophthalmitis ${ }^{15-17}$. However, it is advisable to wait until repair of the laceration has been completed before adding medications because these could be toxic to the retina ${ }^{15-17}$.

Corneal lacerations due to sharp objects have a better prognosis compared to blunt trauma injuries ${ }^{10}$. This is because superficial corneal lacerations may heal completely with medical therapy alone depending on the length of the laceration (less than $15 \mathrm{~mm}$ ). At presentation it is often difficult to assess the visual prognosis and this may lead to the vast majority of injuries requiring primary repair. Most successful procedures performed have resulted in poor vision due to secondary amblyopia and irregular astigmatism. Therefore, correcting refractive error and clearing the visual axis through keratoplasty is important for good visual outcome. A badly injured eye with little possibility of restoring visual potential has very poor prognosis and the visual outcome does not improve 
even after performing several procedures. However, an open globe injury with good visual potential will achieve optimal visual acuity after only about two procedures $^{9,10,18}$. Therefore, it is reassuring that most injuries caused by sharp objects have a fairly good prognosis $10,14,15,17,19$.

\section{Penetrating trauma/Perforating trauma}

A penetrating trauma is a single full thickness wound laceration caused by a sharp object without an exit wound whereas a perforating injury has two full thickness lacerations, an entrance and exit wound caused by the same agent ${ }^{6,12}$. It may be associated with prolapse of the internal contents of the eye ${ }^{24}$. The extent of damage depends on the site of ocular penetration and the momentum of the object at the time of impact ${ }^{4}, 12$.

Penetrating or perforating trauma can occur following an assault, domestic accidents and sports ${ }^{4}, 19,20$. The globe integrity is disrupted by a full-thickness entry wound whereby the eye is pierced by sharp objects such as needles, sticks, pencils, knives, arrows, pens, glass and any object with sharp edges; or by a highvelocity missile such as a piece of metal. The extent of the injury is determined by the size of the object, its speed at the time of impact and its composition. Sharp objects such as a knife cause a well defined laceration of the globe. However, the extent of damage caused by a flying object is determined by its kinetic energy ${ }^{4,19}$.

Wounds affecting only the cornea may not penetrate the anterior segment structures, but may self seal and are less likely to cause visual morbidity, whereas more complex corneal wounds require a healing process which may result in scarring which itself may be visually disabling ${ }^{19}$. Localized or a diffuse lenticular opacity occurs as result from trauma in the anterior segment involving the anterior capsule of the lens. Development of vitreo-retinal traction and scarring occurring after a period from posterior wounds serves as an important factor contributing to the development of complex retinal detachment ${ }^{4,} 19$.

An accurate surgical repositioning of the injured eye is indicated as soon as possible in order to restore normal anatomy while maintaining and controlling the intraocular pressure within normal limits and preventing or controlling infection at the same time $4,11,21$. Enucleation of eyes may be as a result of infection, vitreous abscess, anterior synechiae, cataract and tractional retinal detachment. Further operation might be needed among these complications during the follow-up period ${ }^{21}$. Penetrating injuries require immediate careful attention; an accurate case history to determine how the injury occurred and then prompt surgical repair to prevent functional loss. The major objectives in the management are to relieve pain, preserve or restore vision and to achieve good cosmetic results. If a patient is suspected of having a perforation, intraocular pressure measurements, lids manipulation and motility testing must be avoided. The eye should be covered gently with a sterile gauze or eye pad and protective shield but no pressure should be applied ${ }^{4}$. The risk of intraocular infection is often low but prophylactic antibiotics are routinely prescribed ${ }^{11}$. In any penetrating injury of the globe it is a wise precaution to take X-rays to exclude a retained foreign body ${ }^{11}$.

A penetrating injury of one eye may result in a sympathizing inflammatory reaction in the fellow non-injured eye at any time from two weeks to years later which is an autoimmune disease whereby uveal pigment is released into the bloodstream causing antibodies to be produced resulting in severe uveitis in both the injured and non-injured eye ${ }^{11}$. Risk factors are minimized by removing the injured eye within two weeks if there is no chance of saving useful vision and if the injured eye remains inflamed ${ }^{11}$. The management principles of penetrating ocular injury in children and adults does not differ, but among children difficulty can be encountered during examination and continuing therapy when the child is failing to cooperate 22 . Amblyopia can cause further complications in the treatment of a child. This is often seen in cases when the injury obscures the visual axis (for example, cataracts, corneal scars and vitreous haemorrhages) even for a shorter period of time 22 .

Complications may arise as a result of globe lesions which may depend on the seriousness according to the time of occurrence, method of management and correct assessment ${ }^{21,23}$. In spite of the new microsurgical techniques, the prognosis of penetrating eye injuries in many cases is still quite poor and dependent mostly on the severity $22-24$.

\section{Intra-ocular foreign bodies}

An intra-ocular foreign body (IOFB) is a retained foreign object that enters the eye and may be super- 
ficial or deeply embedded causing an entrance laceration. An IOFB injury is technically a penetrating injury, but due to different clinical implications it is grouped differently because of the treatment modality, timing and rate of endophthalmitis. They are rather variable in presentation, outcome, and prognosis ${ }^{25}$. There are several factors that determine the final resting place and damage caused by an IOFB which include the size, shape and momentum of the object at the time of impact, as well as the site of ocular penetration. Once in the eye, the foreign body may lodge in any of the structures it encounters and may be located anywhere from the anterior chamber to the retina ${ }^{12,25}$.

Intra-ocular foreign body injuries are often due to hammering and metal from grinding equipment or drills that penetrates into the eye. An IOFB may traumatize the eye mechanically, introduce infection or exert other toxic effects on the intraocular structures which may result in inflammation of these structures ${ }^{11,12}$. The risk of infection is relatively low unless the eye is penetrated by vegetable material ${ }^{11}$. Commonly injured structures include the cornea, the lens and the retina ${ }^{25}$.

Retained metallic foreign bodies, especially those containing iron and copper give rise to a serious chemical reaction within the eye ${ }^{11}$. Siderosis involves dissociation of the iron resulting in the deposition of iron in the intraocular epithelial structures including the lens epithelium and retina. Resultant toxic effects include anterior capsular cataract consisting of radial iron deposits on the anterior lens capsule, reddish brown staining of the iris, secondary glaucoma due to trabecular damage and pigmentary retinopathy the last of which has profound effects on vision ${ }^{11,12}$. Chalcosis can also result which is due to an intraocular foreign body with a high copper content causing a violet endophthalmitis like appearance on the Descemet's membrane of the cornea, vitreous and the internal limiting membrane of the retina often with progression to phthisis bulbi ${ }^{11,12}$. Vegetable matter such as cilia may lead to tissue reaction subsequently causing endophthalmitis. Foreign bodies penetrating to the posterior segment may remain suspended in the vitreous or may form a retinal tear or bleeding after striking the retina ${ }^{12,23,24,26 .}$

Initial management, including an accurate case history is vital to determine the origin of the foreign body and it may be helpful for a patient to bring along any causative objects. During the ophthalmic examination special attention is paid to any possible sites of wound entry or exit. Topical fluorescein might be helpful to identify the wound entry and location of the foreign body ${ }^{12}$. However, the best tool to reveal location, size and shape of a foreign body is computed tomography. Gonioscopy can be done if there is suspicion of an IOFB in the angle and retroillumination of the iris may reveal the disruption site by an IOFB. One effective method appears to be prophylactic chorioretinectomy if the IOFB has caused a deep impact involving the choroid. This procedure reduces the risk of post injury proliferative vitreoretinopathy and the initial damage caused at the time of impact.

Planning of surgical intervention involves ruling out the risks of endophthalmitis and subsequent scarring as possible complications ${ }^{27}$. Furthermore, it is vital to determine if the IOFB is anterior or posterior because the surgical approaches will differ ${ }^{27}$. Topical corticosteroids are used to minimize the inflammation and then the IOFB in the anterior chamber is typically removed through a paracentesis but not through the original wound. The IOFB removal is performed $90^{\circ}-180^{\circ}$ from where it is located. Vitrectomy is indicated for posterior segment IOFBs unless tissue damage is minimal ${ }^{27}$. For the actual removal, the best tool to extract a deeply embedded metallic IOFB is a strong intraocular magnet while superficial IOFBs can be removed under slit- lamp visualization using a sterile 26-gauze needle ${ }^{12,27}$. Non-magnetic foreign bodies are removed mechanically with the aid of fine forceps ${ }^{11}$. For large IOFBs, the best way of management is through limbal incision following lensectomy ${ }^{11,12}$.

The prognosis after an IOFB removal is associated with the nature of the injury for example, in the event of blunt trauma, particularly when due to a firearm accident, the prognosis is poor. Advanced techniques for vitreous surgery and instrumentation for IOFB removal under controlled circumstances have brought hope for better results and management of these injuries ${ }^{26,28}$. Intra-ocular foreign body characteristics, particularly size is often the determinant of visual outcome as well as the substance involved and the time of removal. The risk of visual outcome is multiplied by a factor of 1.21 with every $1 \mathrm{~mm}$ increase in the size of the IOFB. Clinical data at presentation also play a vital role in the prognosis e.g.for example, 
the afferent pupil reflex, prolapse of intraocular tissue, lens injury, poor visual acuity and post-operative retinal detachment can have a negative influence on the prognosis ${ }^{26,28}$.

\section{Ruptures}

Globe rupture is a full-thickness wound of the eye wall as a result of contusion or of penetrating trauma on the orbit. Ruptures result in compression of the globe along the anterior-posterior axis resulting in an increase of intraocular pressure to an extent that the sclera tears. Ruptures from blunt trauma occur at the thinnest site of the sclera where the intraocular muscles insert, at the limbus, at the site of previous intraocular surgery and occasionally occurs around the optic nerve. Direct perforation of the globe may be due to sharp objects or those traveling at high velocity. Small foreign bodies may remain within the globe after penetration ${ }^{10,15,19,29}$.

The most common causes of a ruptured globe include blowout fracture of the orbit, sports injuries, occupational injuries, an object thrown from a power tool and an altercation ${ }^{14,20,29}$. The most common symptoms of a ruptured globe include severe eye pain and loss of vision. Additional symptoms of a ruptured globe may include facial swelling, bruising around the eye, double vision, an abnormal pupil, bleeding inside the eye, eye redness and inability to gaze upward with the eye. The uvea, retina, or vitreous may prolapse through the wound $10,15,29$.

A detailed thorough case history must be obtained regarding the mechanism and circumstance of injury from the onset. The possibility of a subdural haemorrhage secondary to falling must be assessed when associated with an extraocular injury. During the assessment of all penetrating and blunt orbital traumas involving objects travelling at high speed, ruling out the possibility of globe rupture should be done first. Examination of the most frequent sites of rupture in the posterior segment is not easily visualized in the presence of superficial injuries. Very small superficial wounds sometimes are difficult to visualize, therefore sharp foreign bodies can enter the eye through these wounds. Protecting the injured eye should be a priority and the ruptured globe should be assessed systematically. Applying too much pressure on the injured eye can cause further damage, therefore should be avoided to prevent potential extrusion of intraocu- lar contents. Young children usually fail to cooperate when trying to evaluate the extent of intraocular injury, however assessment can be carried out under conscious sedation. Identifying an altered reaction of the pupil to light, mydriatic, miotic or eccentric may lead to the realization of a ruptured globe even for a small wound puncture. Treatment may include an eye shield, antibiotics, narcotic pain medications, tetanus immunization and surgery to repair the globe for anterior ruptures by interrupted sutures. If the wound has been extensive and the loss of intra-ocular contents has been great enough that the prognosis for useful function is hopeless, enucleation/evisceration is indicated $^{14-16}$.

A high frequency of permanent visual loss is associated with damage to the posterior segment even though the anatomical position of the globe within the orbit protects the eye from being injured in many situations. Possible complications may involve deep structures of the eye such as infections, delayed postoperative or exogenous endophthalmitis. Within hours endophthalmitis may present after globe rupture or as with fungal organisms depending on the organism involved and the infection may appear weeks later. Maximizing functional outcome is essential with prompt recognition and ophthalmologic intervention. Cases of globe rupture require prompt management by an ophthalmologist $10,15,19$.

Until the appropriate surgical treatment has been performed, the time from injury and the extent of injury determines the visual outcome. Patients should not be given a false impression about their visual outcome until a complete evaluation is done, especially after an operation. Patients presenting with an initial visual acuity of $20 / 200$ or better, $10 \mathrm{~mm}$ or less wound length, anterior wound location inserting to the plane of the four rectii muscles and sharp mechanism of injury, aids with the prediction of excellent final visual acuity (20/60 or better). Predictors of poor vision include initial visual acuity of light perception or no light perception, greater than $10 \mathrm{~mm}$ wound length and wounds from blunt or missile objects extending posterior to the rectii muscles insertion planes. A high frequency of visual impairment is as a result of globe rupture and injury to posterior segment, but with the development of advanced diagnostic techniques, surgical approaches and rehabilitation, the visual outcome can be improved in many eyes ${ }^{10,} 19,29$. 


\section{Closed globe injuries}

Closed globe injuries also occur in everyday life caused by a variety of objects in any environment. However the outcomes and the standard of management following a severe closed globe injury has not been well established especially when associated with vitreous hemorrhages, hence they continue to pose a threat to vision in later stages of injury especially with blunt traumas.

\section{Burns}

Burns to the eye are most often as a result of being in contact with strong acids or alkalis which are amongst the most urgent of ophthalmic emergencies. They are classified by the causative agents involved either chemical injuries, that is, acid, alkali or radiant energy injuries (thermal or ultraviolet). "In particular, the severity of a chemical burn relates to the solution $\mathrm{pH}$, contact duration, solution penetrability and solution quantity"12. Chemical injuries range in severity from trivial to potentially blinding ${ }^{11,12}$. Thermal burns are injuries from radiant energy usually resulting from contact with hot gases, hot liquids, or molten metals ${ }^{11,12}$.

The majority of these injuries are industrial (caustic solutions and solvents), agricultural (fertilizers and pesticides) and the remainder are domestic or households products which can cause complete ocular destruction. Many alkali household products such as drain and floor cleaners should be kept out of the reach of children and be used with extreme caution ${ }^{11,12}$. The most commonly involved alkalis are ammonia, sodium hydroxide and lime while the commonest implicated acids are hydrochloric, sulphuric, chromic and acetic acid $^{11,12}$. Thermal injuries are as a result of being in contact with hot substances such as curling irons, hot curlers, cigarettes and hot liquids.

Alkalis, in particular, cause severe injuries by penetrating the tissues and damaging cells rapidly. Burns primarily damage tissues by coagulating and denaturing cellular proteins and through secondary vascular ischaemic damage ${ }^{30}$. Acid injuries tend to be nonprogressive and superficial. Mild burns usually recover fully even though they result in the loss of the corneal and conjunctival epithelium. Severe burns cause ischaemic damage to the limbal area affecting the vital epithelial stem for subsequent epithelialisation ${ }^{31}$.
After the injury, re-epithelialisation commences, although this may be prevented or hampered by either loss of epithelial stem cells in the limbal region or persistent inflammation. Excessive collagenase activity occurs in the absence of re-epithelialisation leading to progressive corneal thinning and ultimately to perforation of the eye. Visual reduction may be as a result of vascularisation and corneal opacification, tear and mucin deficiencies, necrotic peripheral retinopathy, collagen shrinkage and cataract formation even after epithialisation has occurred ${ }^{30,31}$. Thermal necrosis and penetration can occur from thermal burns however, in thermal burns cell death is limited to the superficial epithelium, whereas ultraviolet burns often have a delayed pain effect and result in punctate keratitis.

In ideal situations, in an eyewash or shower station the affected eye should be irrigated as soon as possible with sterile saline solution to normalize the $\mathrm{pH}$. Further damage to the eye can be reduced by using physiologically balanced solutions for irrigation $^{31}$. To facilitate cooperation a topical anesthetic is applied prior to irrigation or a lid speculum is used to obtain the best irrigation patients eyelids must be opened wide as possible. Initiating copious irrigation at the earliest possible time plays a vital role on the visual outcome. To minimize the damage after a chemical injury, any available neutral fluid can be used to irrigate the eyes immediately for ten minutes (for example, water or soda pop). Following irrigation, presenting to an ophthalmologist is mandatory for a thorough examination ${ }^{12,31,32}$. If an acid burn is suspected, a base should not be used for irrigation in an effort to neutralize the acid. A suitable choice is saline with bicarbonate or lactated ringer balanced solution $^{30,32}$.

Acute chemical injuries are graded to plan an appropriate subsequent treatment and afford an indication of likely ultimate prognosis ${ }^{12,32}$. Grading is performed on the basis of limbal ischaemia severity and corneal clarity. Mild injuries are treated with short course topical steroids, cycloplegics and prophylactic antibiotics for about seven days. The main aims for treatment of more severe burns are to reduce inflammation, prevent corneal ulceration and promote epithelial regeneration ${ }^{12}$. The eye should be patched to establish a conducive environment for re-epithelialization. Emergent ophthalmologic consultation is mandatory for any vision threatening injury be it a 
serious thermal burn or any alkali chemical globe exposure ${ }^{32}$. Early surgery and conservative anti-inflammatory therapy may be necessary to vascularize the limbus, restore the limbal cell population and re-establish the fornices. A procedure known as tenoplasty prevents anterior segment necrosis by re-establishing limbal vascularity and the conjunctival surface in most severe burns ${ }^{30,32}$.

A better understanding of the physiology of the corneal epithelium over the last decade has markedly improved the prognosis of serious forms of ocular burns ${ }^{33}, 34$. Surgical techniques aimed at restoring the destroyed limbal stem cells have also improved the prognosis of severe corneal burns. Prevention, particularly in industry is essential in order to decrease the incidence of burns ${ }^{34}$. On the other hand long term follow up is mandatory to promote the healing process and for providing the best possible opportunity for visual rehabilitation whenever necessary ${ }^{34}$. Advancements in reconstruction of the ocular surface and pathophysiological understanding of a chemical injury or radiant energy has provided hope for patients who would have poor prognosis ${ }^{33}$.

\section{Blunt trauma/Lamella lacerations}

Blunt trauma refers to a direct blow or a type of physical trauma to the eye and surrounding tissues caused by the impact of an object. Damage may occur to anterior segment structures including the eyelid, conjunctiva, sclera, cornea, iris and lens; and posterior segment structures including the retina and optic nerve resulting in significant visual loss ${ }^{35}$, 36. It includes contusions and lamellar laceration of the globe. A contusional injury has no (full thickness) wound. The injury is either due to choroidal rupture (direct delivery of the object) or angle recession (changes in the shape of the globe). Lamellar laceration is a partial thickness wound of the eyewall caused by a sharp object. Both structural and functional damage to the eye can be as a result of blunt trauma ${ }^{36}$. Traumatic macular holes and retinal detachment or dialysis may also occur after blunt ocular trauma ${ }^{37-39}$. In the uncommon situation when force is applied directly to the overlying sclera the posterior segment can be damaged, although blunt injuries may also damage the posterior pole of the eye ${ }^{40}$. Retinal detachment after blunt trauma may develop as a result of retinal dialysis, flap tears, or giant retinal tears through rapid compression-decompression forces that result in transient anteroposterior shortening and equatorial elongation of the globe ${ }^{37}$.

The most common cause of blunt trauma is the eye being struck with a finger, fist, racket, tennis ball, or other solid object. At the moment of impact sudden compression and indentation of the globe occurs producing damage to the eye ${ }^{36}$.

Blood occupying the inferior part of anterior chamber is often observed, a condition referred to as a hyphaema ${ }^{4}$. Prolonged unresolved hyphaemia and corneal blood staining usually cause peripheral anterior synechiae. Normally damage to the crystalline lens forms a cataract preventing light from reaching the retina, or it may no longer be able to focus clear images due to displacement within the eye ${ }^{4}$. Blunt trauma can damage the retina as well. The presence of a tear in the retina leading to large retinal detachment may result in reduced vision whenever the area on the retina responsible for clear vision is affected ${ }^{41}$. In some instances, sudden effects may not be evident until months or years later after the injury occurs such as a cataract, retinal detachment, or glaucoma and all of these may result in visual loss ${ }^{4}$.

Any hyphaema present should be examined by an ophthalmologist as soon as possible to encourage the blood to settle which usually involves bed rest with the head of the bed elevated. Anesthesia is indicated for a thorough evaluation of the peripheral retina. Atropine may be given to dilate the pupil and corticosteroids to reduce inflammation within the eye. Further injury can be prevented with the use of a protective shield taped over the eye. Patients with blunt eye trauma should be under steady observation by an ophthalmologist to handle late complications such as proliferative vitreoretinopathy which is a condition that generally results from chronic retinal detachment or retinal surgery and severe ocular trauma ${ }^{36}$. Prompt surgical repair is required for retinal detachments to prevent or to minimize serious visual loss. Follow-up care must be continuous to preserve vision after an injury by monitoring the intraocular pressure at least once daily for the first few days because if the pressure is elevated it may result in secondary open-angle glaucoma ${ }^{41}$. For children who sustain severe injuries enough to result in a hyphaema are urged to go for annual examinations because children may not recognize decreased vision at an early stage and present 
when visual loss is severe ${ }^{41}$.

For blunt traumas, the prognosis is dependent on the original injury rather than the complications as a result of hyphaema formation. After initial injury the risk duration for certain complications remains unknown, however, as a result of retinal surgery, severe ocular trauma and chronic retinal detachment, proliferative vitreoretinopathy is one possible complication. Poor visual outcome is expected during vitreoretinal surface traction created by proliferative membranes, causing further complications and visual loss even though the nidus for proliferation is unclear ${ }^{42}$. A high percentage of patients with injuries confined to the posterior segment achieve good standard of vision initially, but a significant number of patients lose all useful vision at a later stage several weeks after injury due to the development of a tractional retinal detachment $2,39,40,42$.

\section{Conclusion}

The eye remains a high risk organ for ocular injuries resulting in visual impairment or blindness. Patients continue to lose eyes through enucleation or evisceration despite the high quality of surgical service after eye trauma and secondary surgical procedures. Blunt mechanisms have poor visual outcome while sharp injuries have good prognoses in this respect. Clinically the predictor of visual outcome is the location of a posterior wound. Irreversible damage to the optic nerve and retina can occur following a posterior segment injury even after the structural integrity of the globe has been repaired and thus carries a poor visual prognosis $37,40,43$. It is reassuring that immediate presentation to the eye clinic may improve the prognoses, however, in some cases it may remain poor. Prevention of eye injuries cannot be over emphasized and this is a combined responsibility of ophthalmologists, optometrists, pediatricians, parents, teachers and coaches.

\section{References}

1. Cockerham GC. Blunt trauma and nonpenetrating injuries of the anterior segment. Ophthalmol 198390 140-148.

2. Eagling EM. Perforating injuries of the eye. Br J Ophthalmol 197660 732-736.

3. Gyasi ME, Adjuik MA, Amoaku, WMK. Epidemiology of hospitalized ocular injuries in the Upper East Region of
Ghana. Ghana Med J 2007 41(4) 171-175.

4. MacGwin G, Owsley C, Xie A. Rate of eye injury in the United States. Arch Ophthalmol 2005123 970-976.

5. Juthani V, Bruce M. The epidemiology and incidence of visual deficits following ocular trauma in pediatric patients. Invest Ophthalmol Vis Sci 20072 1335-1338.

6. Kuhn F, Morris R, Witherspoon CD, Heimann K, Jeffers JB and Treister G. A standardized classification of ocular trauma. Graefes Arch Clin Exp Ophthalmol 1996 234(6) 399-403.

7. Kuhn F, Pieramici DJ. Ocular trauma: principles and practice. New York, Thieme, 2002 236-246.

8. Schrader WF. Open Globe Injuries: Epidemiological Study of Two Eye Clinics in Germany, 1981-1999. Croat Med J 200445 268-274.

9. Pieramici DJ, MacCumber MW, Humayun MU, Marsh MJ, de Juan E Jr. Open globe injury: update on types of injuries and visual results. Ophthalmol 1996103 1798-803.

10. McGowan Jr. G, Hall TA, Xie A, Owsley C. Trends in eye injury in the United States, 1992-2001. Invest Ophthalmol Vis Sci 2006 47(2) 521-527.

11. Coakes R, Sellers PH. Outline of ophthalmology. (2nd edition) Oxford: Butterworth Heinemann, 1995 130-165.

12. Kanski JJ. Clinical ophthalmology. A systemic approach. $\mathrm{St}$ Louis: Butterworth Heinemann, 2003, pp665-680.

13. Chiapella AP, Rosenthal AR. One year in an eye casualty clinic. Br J Ophthalmol 1985 69(11) 865-70.

14. Barr CC. Prognostic Factors in Corneoscleral Lacerations. Arch Ophthalmol 1983 101(6) 919-924.

15. Rahman I, Maino A, Devadason D and Leatherbarrow B. Open globe injuries: factors predictive of poor outcome. Eye 200620 1336-1341.

16. Sanders N. Repair of corneal lacerations. Ann Ophthalmol 19757 (11) 1515-1520.

17. Naidu K . The injured eye; practical management guidelines and referral criteria for the rural doctor. SA Fam Pract 2006 48(7) 39-45.

18. Parver LM, Dannenberg AL, Blacklow B, Fowler CJ, Brechner RJ, and Tielsch JM. Characteristics and causes of penetrating eye injuries reported to the National Eye Trauma System Registry, 1985-91. Public Health Rep 1993 108(5) 625-632.

19. Patockova A, Stermen P, Krasnik V, Olah Z. Mechanical injuries of the eye. Bratisl Med J 2010 111(6) 329-335.

20. Napier SM, Baker RS, David G. Sanford DG, Easterbrook M. Eye injuries in athletics and recreation. Surv Ophthalmol 1996 41(3) 229-244.

21. Adhikary HP, Tailor P and Fitzmaurice DJ. Prognosis of perforating eye injury. Br J Ophthalmol 197660 737-739.

22. Thompson CG, Kumar N, Billson FA, Martin F. The aetiology of perforating ocular injuries in children. Br J Ophthalmol 200286 920-922.

23. Mackiewicz J, Matejko EM, Pylak MS, Sidor MP, Zagórski Z. Work related, penetrating eye injuries in rural environments. Ann Agric Environ Med 200512 27-29.

24. Smith D, Stack LB, Wrenn K. The epidemiology and diagnosis of penetrating eye injuries. Acad Emerg Med 20029 
209-213.

25. Imrie FR, Cox A, Foot B, MaCewen CJ. Surveillance of intraocular foreign bodies in the UK. Eye 2008 22(9) 11411147.

26. Szijártó Z, Gaál V, Kovács B, Kuhn F. Prognosis of penetrating eye injuries with posterior segment intraocular foreign body. Arch Clin Exp Ophthalmol 2008246 161-165.

27. Kuhn F, Cooke CA. A closer look at anterior segment intraocular foreign bodies. Eye 2005 19(4) 476-478.

28. Roy FH, Fraunfelder FW, Fraunfelder FT. Roy and Fraunfelder's current ocular therapy (6th edition). USA: Elsevier, 2007 288-290.

29. Doyle J. Patient options after a ruptured globe. J Ophthal Med Technol 2009 5(2) 5.

30. Kuckelkorn R, Keller G, Redbrake C, Schrage N. Emergency treatment of chemical and thermal eye burns. Acta Ophthalmol Scand 2002 80(1) 4-10.

31. Wagoner MD. Chemical injuries of the eye: current concepts in pathophysiology and therapy. Surv Ophthalmol 1997 41(4) 275-313.

32. Ikeda N, Hayasaka S, Hayasaka Y, Watanabe K. Alkali burns of the eye: effect of immediate copious irrigation with tap water on their severity. Ophthalmol 2006 220(4) 225-228.

33. Fish R, Davidson RS. Management of ocular thermal and chemical injuries, including amniotic membrane therapy. Curr Opin Ophthalmol 2010 21(4) 317-322.

34. Khodabukus R, Tallouzi M. Chemical eye injuries: presentation, clinical features, treatment and prognosis. Nurs Times 2009 105(22) 28-29.

35. Meallet MA. Trauma to the orbit: Current therapy of trauma and surgical critical care. California, Elservier, 1994 181-196.

36. Viestenz A, Küchle M. Blunt ocular trauma. Part 21. Adhikary HP, Tailor P and Fitzmaurice DJ. Prognosis of perforating eye injury. Br J Ophthalmol $1976 \mathbf{6 0}$ 737-739.

37. Dadgostar H, Ventura ACM and Hayden BC. Posterior Segment Trauma. Ophthalmol 2008 3(2) 267-272.

38. Williams DF, Mieler WF, Williams GA. Posterior segment manifestations of ocular trauma. Retina 1990 109(1) 35-44.

39. Meier P. Combined anterior and posterior segment injuries in children: a review. Graefes Arch Clin Exp Ophthalmol 2010248 1207-1219.

40. Canavan YM, O’Flaherty MJ, Archer DB, Elwood JH. A 10-year survey of eye injuries in Northern Ireland. $\mathrm{Br} \mathrm{J}$ Ophthalmol 198064 618-625.

41. Kaushik S, Sukhija J. Blunt ocular trauma. Ann Ophthalmol 2006 38(3) 249-252.

42. Waters T, Vollmer L, Sowka J. Proliferation vitreoretinopathy as a late complication of blunt ocular trauma. J Am Optom Assoc 2008 79(4) 197-202.

43. Abraham D, Isseme I, Vitale S, West S. Epidemiology of eye injuries in rural Tanzania. Ophthalmic Epidemiol 1999 6(2) 85-94. 